In our first retrospective audit of fifteen obstetric women admitted to ITU over a period of two years, services were assessed using CNST standards 2.9, 4.8, 5.10. This audit highlighted poor documentation and difficulty in identifying evidence. Recommendations were made including defined auditable standards and a named person to ensure compliance. Local guidelines were revised. From recommendations we identified twelve auditable standards. Documentation of clearly defined reason for transfer to ITU, daily multidisciplinary review, SBAR (situation, background, assessment, recommendation) handover, entry level of ITU, length of stay, discharge criteria, outreach follow up and clinical incident form were assessed.

A repeat audit of eight obstetric ITU admissions in next twelve months was completed. Retrospective case notes review was conducted by the same person. Re audit confirmed improved documentation however extracting evidence continued to be difficult.

Numerous national guidance and standards can be confusing however it is possible to identify local auditable standards to improve care and assessment of care.

\section{PM.56 AUDIT OF CARE OF CRITICALLY ILL PREGNANT WOMEN}

doi:10.1136/archdischild-2013-303966.138

A Ram Mohan, U Ihekwaba, E Jwarah. Milton Keynes NHS Foundation Trust, Milton Keynes, UK

Aim To review series of critically ill obstetric patients admitted to ITU and to formulate a guideline for the care of these women.

Background The women who become critically ill during pregnancy should receive the same standard of care for both their pregnancy related and critical care needs, delivered by professionals with the same level of competences irrespective of whether these are provided in a maternity or general critical care setting. ${ }^{1}$

Methods Retrospective study of 55 women who were admitted to critical care unit from $01 / 01 / 2006$ to $31 / 12 / 2011$. Patients were identified by ITU database.

Results Average ITU stay was $1-2$ days in $50 \%$ of cases. $92 \%$ of patients were admitted postpartum. Massive obstetric haemorrhage $(54 \%)$, sepsis (13\%), Pre eclampsia/HELLP/Eclampsia (11\%) and swine flu $(5.4 \%)$ were the main indications. $55 \%$ of the patients were mechanically ventilated.100\% compliance with MEWS chart was observed. The most common interventions were arterial line (64\%) and CVP line (35\%). VTE assessment on admission to ITU was observed in $65 \%$, daltaparin (74\%) and TEDS (74\%) of cases. One case of group A streptococcus was seen. Maternal mortality was nil. Debriefing of the family (61\%) and debriefing of patient $(78 \%)$ cases. Datix completed (10\%), external transfer $(5.4 \%)$ cases.

Conclusions Massive obstetric haemorrhage, sepsis and pre eclampsia are the main reasons for admissions.

Recommendations Documentation of patient and family debrief needs to be improved. All these women should be seen in gynaecology follow up clinic for debriefing. Guidelines for critically ill pregnant or recently pregnant women and sepsis in pregnancy and puerperium should be formulated.

\section{REFERENCE}

1. Providing equity of critical and maternity care for the critically ill pregnant or recently pregnant woman, July 2011, Joint RCOG guideline.

\section{PM.57 REFRACTORY SVT IN THE THIRD TRIMESTER OF PREGNANCY: MANAGEMENT DILEMIMAS}

doi:10.1136/archdischild-2013-303966.139

K Merrick, S Maydanovych, U Rajesh. York Teaching Hospital, York, UK
The most frequently encountered arrhythmia, commonest in women of reproductive age, is paroxysmal supraventricular tachycardia (SVT). Atrio-ventricular nodal re-entry and Wolf-ParkinsonWhite syndrome account for the majority of these (1). Evidence for treatment during pregnancy is scarce due to the lack of research in this group of patients and limited information on the safety of antiarrhythmic drugs in pregnancy. Much of the current evidence is based on case reports, animal studies, observational studies and clinical experience however, several methods appear to be reasonably safe for the patient and the fetus (2). There is limited evidence regarding the safety and use of DC cardioversion in pregnancy $(3,4)$. Particular consideration is required to be given to the gestation and the risks of delivery when considering the various treatments including choice of routinely used first to third line antiarrhythmic agents and DC cardioversion for the more refractory situations. We describe a case of refractory SVT in a patient with a failed ablation for WPW syndrome presenting in the third trimester of pregnancy. This case highlights several management dilemmas including decisions regarding choice and dose of pharmacological agents, planning a Caesarean section for delivery of fetus prior to DC cardioversion that was required and particularly emphasises good practise with a multidisciplinary team approach at every stage of the management process.

\section{REFERENCES}

1. Lee SH, Chen SA, Wu TJ et al, Effects of pregnancy on first onset and symptoms of paroxysmal supraventricular tachycardia. Am J Cardiol 1995;76:675.

2. Joglar J.A., Page R.L.; Treatment of cardiac arrhythmias during pregnancy: safety considerations. Drug Saf 20 1999;85-94.

3. Klepper I. Cardioversion in late pregnancy. Anaesthesia 1981;36:611-6.

4. Finlay AY, Edmunds V. DC cardrioversion in pregnancy. Br J Clin Pract 1979;33: 88-94.

\section{PM.58 CLINICAL OUTCOMES IN PREGNANT WOMEN NEWLY RECLASSIFIED AS GESTATIONAL DIABETES (GDM) USING IADPSG CRITERIA}

doi:10.1136/archdischild-2013-303966.140

R Anthony, A lkomi, R Khan, P Angala, S Kiss. Basildon and Thurrock university hospital, Basildon, Essex, UK

Background There are widespread concerns about the potential resource implications of implementing the International Association of the Diabetes and Pregnancy Study Group (IADPSG) criteria for diagnosing GDM. However, another important consideration is the risks facing undiagnosed cases if the new criteria are not adopted. In our unit, since 2006 we have offered a 3 point OGTT [fasting, $1 \& 2$ hours]. Hence our projected increase in number of GDM cases is solely related to the lower IADPSG fasting threshold [5.1] and not it's addition of a $1 \mathrm{hr}$ threshold.

Aims To identify women with 75 g OGTT fasting levels between IADSPG and WHO thresholds [5.1-5.9], and normal 1-2 hour levels. To ascertain the extent of diabetes related outcomes in these undiagnosed cases.

Materials and Methods Retrospective study of OGTT results for all women who delivered in our unit between $1^{\text {st }}$ January 2009 and 31 $1^{\text {st }}$ December 2011. Outcome data for 129 selected cases was obtained from our Protos Maternity information system.

Results All deliveries ended in live births but the fetal macrosomia rate $(>4 \mathrm{~kg})$ was $30 \%$ [39/129]. Using a cut off of $4.5 \mathrm{~kg}$ the macrosomia rate was $5.4 \%$. The caesarean section rate for macrosomic babies was $23 \%$ [9/39]. Neonatal hypoglycaemia was diagnosed in $6.2 \%$ [4/129] of the babies and 3.1\% [4/129] needed immediate admission to NICU. However, none of the admissions to NICU were directly related to neonatal hypoglycaemia. 